\title{
Atrial fibrillation and coronary stenting: is the AUGUSTUS trial the end of triple therapy?
}

\author{
Bastiaan Zwart ${ }^{1}$ \& Jurriën M Ten Berg*,1 \\ ${ }^{1}$ Department of Cardiology, St Antonius Hospital, Nieuwegein, The Netherlands \\ *Author for correspondence: j.ten.berg@antoniusziekenhuis.nl
}
"'Therefore, the question whether (subgroups of) patients at high ischemic risk might still benefit from triple therapy remains unanswered."

First draft submitted: 20 May 2019; Accepted for publication: 28 May 2019; Published online: 6 August 2019

Keywords: acute coronary syndrome $\bullet$ atrial fibrillation $\bullet$ AUGUSTUS • nonvitamin K oral anticoagulant $\bullet$ oral anticoagulant • percutaneous coronary intervention • triple therapy $\bullet$ vitamin $\mathrm{K}$ antagonist

Patients with atrial fibrillation (AF) on oral anticoagulation (OAC) who undergo coronary stenting or suffer acute coronary syndrome (ACS) have posed a challenge for clinicians for many years now. As one in five patients with $\mathrm{AF}$ undergo percutaneous coronary intervention $(\mathrm{PCI})$ at some point in life, it is clear that this is a relevant issue in daily clinical practice [1]. From the PCI perspective, 1 in 12 patients undergoing coronary stenting has concomitant $\mathrm{AF}$ and an indication for oral anticoagulation [2]. For these patients, both anticoagulations are indicated in order to prevent systemic embolism including cerebrovascular accident, whereas dual antiplatelet therapy has been shown to be very effective in preventing stent thrombosis and recurrent myocardial infarction $[3,4]$.

Triple therapy is effective from both points of view but is associated with a two- to three-fold increase in bleeding [4]. Therefore, it was hypothesized that the combination of OAC and a P2Y12-inhibitor with the omittance of aspirin might be effective in preventing ischemic events while reducing bleeding. Until now, only three trials investigated such a dual therapy strategy. The WOEST trial was the first to assess a regimen of dual therapy (vitamin K antagonist [VKA] plus clopidogrel) versus triple therapy (VKA plus clopidogrel and aspirin) and demonstrated that dual therapy was safe in ischemic terms and significantly reduced bleeding [5]. The WOEST trial was, however, seriously underpowered for the ischemic end point. Meanwhile, the world of antiplatelet and antithrombotic agents changed with the introduction of more potent P2Y12-inhibitors including prasugrel and ticagrelor and with the introduction of the of nonvitamin $\mathrm{K}$ oral anticoagulant therapy (NOAC). Subsequently, two studies investigated the combination dual therapy with NOAC plus a P2Y12-inhibitor with a triple therapy strategy including VKA, a P2Y12-inhibitor and aspirin [6,7]. Both studies demonstrated a lower risk of bleeding associated with the former regimen, but due to the design of these studies, it was not clear whether this was an effect of omitting aspirin, a benefit of NOAC over VKA or an effect of the lower NOAC dose used in the studies. Furthermore, ticagrelor and prasugrel were infrequently used (i.e., $12 \%$ of patients in RE-DUAL PCI $[6]$ and a $<10 \%$ in PIONEER-AF [7]). Finally, the main issue with all three studies was insufficient power for the ischemic end point including stent thrombosis.

But now we have the long-awaited results of AUGUSTUS. The trial assessed the efficacy and safety in an elegant $2 \times 2$ design comparing the use of VKA versus NOAC and aspirin versus placebo. This largest study thus far included 4614 patients with ACS or who underwent PCI. In short, the study demonstrated that a combination of full dose apixaban with a P2Y12-inhibitor but without aspirin resulted in less bleeding compared with regimens that included a VKA with a P2Y12 inhibitor. Dropping aspirin was without a penalty in terms of ischemic events [8]. First of all, it is important to notice that again this study established the benefits of NOAC over VKA which now also holds true in patients with concomitant (dual) antiplatelet therapy. Based on their favorable safety profile, NOAC should be preferred over VKA, also in combination with antiplatelet agents. Second, the evidence is strongly in favor 
of double therapy (dropping aspirin after the procedure or at discharge). But is this the end of triple therapy? Hold your horses. A few methodological and conceptual issues need attention. First of all, the majority of AUGUSTUS patients (>60\% in all treatment arms) had either medically managed ACS or underwent elective PCI. One could argue that these patients are not the real high-risk patients at risk for recurrent ischemic events. Second, the study was not powered for ischemic events and a numerical higher incidence of stent thrombosis was observed in patients not receiving aspirin. Third, more than $90 \%$ of patients in all treatment arms received clopidogrel and not one of the more potent P2Y12-inhibitors thus leaving the question unanswered how NOAC's behave in combination with ticagrelor (or prasugrel). And last but not least, the median time to randomization was 6 days, implying that $50 \%$ of patients received aspirin for a week after the index event or PCI. Interestingly, a 2018 North American focused update on the topic endorsed such a strategy of in-hospital triple therapy as a default strategy in our patient category [9]. The most recent ESC guideline on revascularization on the other hand, published around the same time, still advocates a triple therapy for 1 month in most patients unless concerns about bleeding risk prevail [4].

But the main issue with all of the aforementioned studies is this: 'one size does not fit all'. All studies so far fail to make a distinction between high-risk and low-risk patients. For example, a 50-year old patient undergoing elective 1 -vessel PCI is very different from a 70-year old patient with multivessel PCI, previous myocardial infarction and substantial comorbidity such as diabetes. Indeed, AF patients undergoing PCI constitute a very heterogenous group in whom individual risk might be dominated by high bleeding risk, high risk for recurrent thrombotic events or both. Importantly, The ADAPT-DES study already showed that bleeding is not a benign side effect of antithrombotic therapy but is a serious adverse event which is associated with similar or even higher mortality rates as compared with recurrent myocardial infarction [10].

In the field of duration of dual antiplatelet therapy (DAPT), recently attention has focused on individualization and tailoring treatment for every patient. Two relevant risk scores are currently recommended to use in determining optimal duration of DAPT. First, bleeding risk should be estimated after PCI in order to identify patients at high bleeding risk in whom the risks-benefit ratio might favor reduced DAPT duration. The PRECISE-DAPT score is the only validated risk score for estimating bleeding risk after PCI [11]. Subsequently, in patients not at high bleeding risk and who tolerate the first course of DAPT well (i.e., no significant bleeding events during follow-up), the DAPT score can be applied selecting patients who might benefit from prolonged DAPT duration up to 30 months [12].

Unfortunately, no risk scores yet exist to determine optimal treatment for AF patients undergoing PCI. Although the results of the AUGUSTUS trial suggest that double therapy is the new default strategy for low risk patients, patients at high ischemic risk were under-represented. Therefore, the question whether (subgroups of) patients at high ischemic risk might still benefit from triple therapy remains unanswered. Future research into this field should be aimed at identifying risk subgroups in order to enable tailored therapy in AF patients undergoing PCI.

Financial \& competing interests disclosure

B Zwart receives speakers fees from Astra Zeneca and Bayer. The authors have no other relevant affiliations or financial involvement with any organization or entity with a financial interest in or financial conflict with the subject matter or materials discussed in the manuscript apart from those disclosed.

No writing assistance was utilized in the production of this manuscript.

\section{References}

1. Mehta SR. Refining antithrombotic therapy for atrial fibrillation and acute coronary syndromes or PCI. N. Engl. J. Med. 380(16), 1580-1581 (2019).

2. Valgimigli M, Bueno H, Byrne RA et al. 2017 ESC focused update on dual antiplatelet therapy in coronary artery disease developed in collaboration with EACTS: the task force for dual antiplatelet therapy in coronary artery disease of the European Society of Cardiology (ESC) and of the European Association for Cardio-Thoracic Surgery (EACTS). Eur. Heart J. 39(3), 213-260 (2018).

3. Kirchhof P, Benussi S, Kotecha D et al. 2016 ESC Guidelines for the management of atrial fibrillation developed in collaboration with EACTS. Europace 18(11), 1609-1678 (2016).

4. Neumann F, Sousa-Uva M, Ahlsson A et al. 2018 ESC/EACTS Guidelines on myocardial revascularization. Eur. Heart J. 40(2), 87-165 (2018).

5. Dewilde WJM, Oirbans T, Verheugt FWA et al. Use of clopidogrel with or without aspirin in patients taking oral anticoagulant therapy and undergoing percutaneous coronary intervention: an open-label, randomised, controlled trial. Lancet 381(9872), 1107-1115 (2013). 
6. Cannon CP, Bhatt DL, Oldgren J et al. Dual antithrombotic therapy with dabigatran after PCI in atrial fibrillation. N. Engl. J. Med. 377(16), 1513-1524 (2017).

7. Gibson CM, Mehran R, Bode C et al. Prevention of bleeding in patients with atrial fibrillation undergoing PCI. $N$. Engl. J. Med. 375(25), 2423-2434 (2016).

8. Lopes RD, Heizer G, Aronson R et al. Antithrombotic therapy after acute coronary syndrome or PCI in atrial fibrillation. N. Engl. J. Med. 380(16), 1509-1524 (2019).

9. Angiolillo DJ, Goodman SG, Bhatt DL et al. Antithrombotic therapy in patients with atrial fibrillation treated with oral anticoagulation undergoing percutaneous coronary intervention. Circulation 138(5), 527-536 (2018).

10. Généreux P, Giustino G, Witzenbichler B et al. Incidence, predictors, and impact of post-discharge bleeding after percutaneous coronary intervention. J. Am. Coll. Cardiol. 66(9), 1036-1045 (2015).

11. Costa F, van Klaveren D, James $S$ et al. Derivation and validation of the predicting bleeding complications in patients undergoing stent implantation and subsequent dual antiplatelet therapy (PRECISE-DAPT) score: a pooled analysis of individual-patient datasets from clinical trials. Lancet 389(10073), 1025-1034 (2017).

12. Yeh RW, Secemsky EA, Kereiakes DJ et al. Development and validation of a prediction rule for benefit and harm of dual antiplatelet therapy beyond 1 year after percutaneous coronary intervention. JAMA 315(16), 1735-1749 (2016). 
